\title{
Pyrrolidinyl Group as Charge Donor for the Excited State Intramolecular Chargetransferin (pyrrolidin-1-yl) phenyl) Prop-2-en-1-one 3-(4-methoxyphenyl)-1-(4-
}

\author{
Nidhi Mishra ${ }^{1, *}$, Kamal Kumar Chaudhary $^{1}$ and Satish Kumar Awasthi ${ }^{2}$ \\ ${ }^{1}$ Division of Applied Sciences \& IRCB, Indian Institute of Information Technology, Allahabad, Deoghat, \\ Jhalwa, U.P. - 211012, India \\ ${ }^{2}$ Chemical Biological Laboratory, Department of Chemistry, University of Delhi-110007, India
}

\begin{abstract}
The absorption and steady state emission properties of a chemically synthesized chalcone, 3-(4methoxyphenyl)-1-(4-(pyrrolidin-1-yl) phenyl) prop-2-en-1-one (MPPP) containing asymmetrical donor and acceptor groups has been investigated both experimentally and theoretically. The ground state, MPPP has a significant intramolecular charge transfer (ICT) character and a great sensitivity to the hydrogen bond donating ability of the medium as reflected from the absorption spectra in pure non polar, polar and neutral solvents. On the other hand, its excited singlet state exhibits high ICT characters as manifested by the drastic solvatochromic effects. These results are consistent with the data. The absorption spectra of the compound MPPP undergoes minor changes with increasing polarity of the solvents and the fluorescence spectra experiences a distinct bathochromic shifts in the both position and fluorescence quantum yields, increases reaching a maximum before decrease with increasing the solvent effects. The quantum yields decrease with increase in the solvent polarity. The magnitude of change in the dipole moment was also calculated using Austin Model 1 (AM1). These results suggest that the evidence about the intramolecular charge transfer in the emitting singlet state of this compound. The solvent dependence of quantum yields of MPPP was interpreted on the basis of positive and negative solvatokinetic as well as hydrogen bonding effects. Intramolecular charge (ICT) transfer took place from pyrrolidine nitrogen to $\alpha, \beta$ unsaturated carbonyl in the ground state.
\end{abstract}

Keywords: Chalcone, Solvatokinetic, Intramolecular charge transfer, Dipole moment, AM1 method.

\section{INTRODUCTION}

The photophysical properties of organic molecules has achieved a significant importance in recent times owing the wide applications in various fields [1]. Fluorescence spectroscopy is the most widely used technique in the area of cellular biology and biomedical to understand the microenvironment of molecular assemblies of macromolecules and more recently even in pollution control [2, 3]. Although, large number of fluorescent molecules has been developed yet search is still under way. The fluorophore thus developed should have easy to synthesize and good photophysical properties because on photoexcitation, redistribution of electronic charges at each atom (especially on the acid and basic centers) of the molecule does take place. Moreover, on photoexcitation, fluorophores containing both an electron donor and an electron acceptor exhibit an internal charge-transfer (ICT) state or twisted internal charge transfer (TICT) state [4]. Thus, the organic molecule having intramolecular charge transfer (ICT) properties are of continuing theoretical and experimental interest.

*Address correspondence to this author at the Division of Applied Sciences \& IRCB, Indian Institute of Information Technology, Allahabad, Deoghat, Jhalwa, U.P. - 211012, India; Tel: +91-532 -2922540; Fax: +91-532- 2430006;

E-mail: nidhimishra@iiita.ac.in
Chalcones, the biochromophorioc molecules separated by a keto-vinyl chain, constitute an important class of privileged structure that has a wide range of biological properties [5]. Chalcones are widely used for various optical applications such as optical substances, photorefractive polymers [6], holographic recording materials and fluorescent probes for sensing of metal ions $[7,8]$, biological macromolecules $[9,10]$, and microenvironment in micelles [11]. Therefore, studies of the photophysical properties of chalcones have significant importance [12-15]. In the last decades, several studies were done on substituted enone derivatives with different bridge structures in various polar solvents because substituted enone derivatives are very effective photoinitiators. Various groups have studied photophysical properties of substituted chalcones involving mostly asymmetric donor-acceptor (D/A) chalcones [16] and to a lesser extent symmetrical donor acceptor chalcones [17, 18]. In such system, carbonyl group acts as acceptor while substituted phenyl group act as donor. Such D/A molecules are good example in which very large changes in charge distribution can be induced in the excited state upon absorption of light photons. This phenonmena leads to large generation of dipole (due to photoinduced intramolecular charge transfer, ICT). This results in a strong interaction with the surrounding medium to cause not only solvent reorganization but also some time to structural rearrangement in the solute itself. 
Our research effort is focused on synthesis, characterization and evaluation of various substituted chalcones for their antimalarial and antifilarial activity $[19,20]$. To diversifying our research interest, we initiated photophysical properties of some of the chalcones synthesized in our laboratory. We choose bichromophoric systems for the study of photoinduced electron transfer which has an immense application in the area of photophysics, photochemistry, polymer physics, optics technology and laser physics [21]. We choose three chalcones viz. 3-(4-Chloro-phenyl)-1-(4pyrazol-1-yl-phenyl)- propenone (CPPP-1), 3-(4Chloro-phenyl)-1-(4-pyrrolidin-1-yl-phenyl)-propenone (CPPP-2), 3-(4-Methoxy-phenyl)-1-(4-pyrrolidin-1-ylphenyl)-propenone (MPPP), for fluorescence studies (Figure 1a). Among all three, only one compound MPPP had shown fluorescence. In this paper, we described the ground state and excited state intramolecular charge transfer (ICT) characteristics of 3-(4-methoxyphenyl)-1-(4-(pyrrolidin-1-yl) phenyl) prop- 2-en-1-one (MPPP) (Figure 1b) in various solvents containing donor and acceptor in the same molecule. The optimized molecular structure is shown in (Figure 1c). We have used experimental and theoretical methods.

\section{EXPERIMENTAL DETAILS}

\subsection{Materials and Methods}

Synthesis and characterization of the MPPP is reported elsewhere $[19,20]$. Briefly, the compound 3(4-methoxyphenyl)-1-(4-(pyrrolidin-1-yl) phenyl) prop-2en-1-one was synthesized by the Cliasen-Smith condensation procedure. To a solution of 4fluoroacetophenone $(5.12 \mathrm{gm}, 40 \mathrm{mmol})$ in $5 \mathrm{ml}$ anhydrous DMF, pyrrolidine $(2.84 \mathrm{gm}, 40 \mathrm{mmol}$ ) and $\mathrm{K}_{2} \mathrm{CO}_{3}(6.9 \mathrm{gm}, 50 \mathrm{mmol})$ were added. The reaction mixture was refluxed for $18 \mathrm{hs}$ at $110{ }^{\circ} \mathrm{C}$. On completion, as checked by thin layer chromatography (TLC), the DMF was evaporated in vacuo and re-<smiles>COc1ccc(/C=C/C(=O)c2ccc(-n3cccn3)cc2)cc1</smiles><smiles>CC=N</smiles><smiles>COc1ccc(/C=C/C(=O)C2=CCC(=[N+]3CCCC3)C=C2)cc1</smiles>

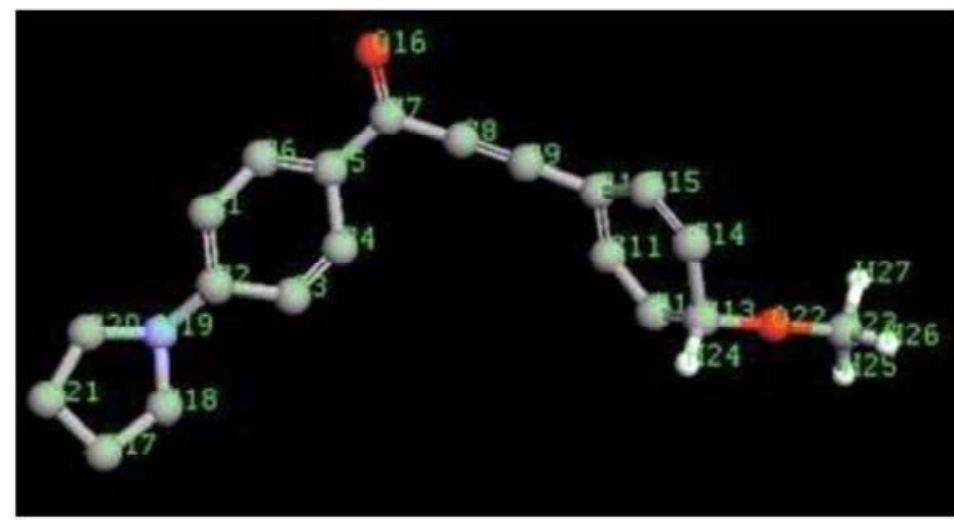

Figure 1: a: Structure of various chalcone analogs.

b: Molecular structure and intramolecular charge transfer process in MPP.

c: Optimized molecular structure of MPPP by AM1 method using Argus Lab software. 
dissolved into water $(50 \mathrm{ml})$. The aqueous solution was extracted with chloroform $(3 \times 50 \mathrm{ml})$ and the combined organic solution was dried over anhydrous $\mathrm{Na}_{2} \mathrm{SO}_{4}$ and evaporated in vacuo to yield crude 4-pyrrolidine acetophenone. The intermediate was purified by flash column chromatography and characterized by mass (Macromass G spectrophotometer) and NMR prior to use in the next step. The remaining other two intermediates were prepared by the similar method.

To a stirred solution of column purified 4-pyrrolidine acetophenone $(1.13 \mathrm{gm}, 6 \mathrm{mmol})$ and 4-methxoy benzaldehyde $(0.816 \mathrm{gm}, 6 \mathrm{mmol})$ in a minimum amount of methanol (normally $10 \mathrm{ml}$ ), $\mathrm{NaOH}$ pellets $(600 \mathrm{mg})$ were added at $0{ }^{\circ} \mathrm{C}$. The reaction mixture was allowed to draw closer to room temperature and stirred for 18-20 hrs. Appearance of off-white to yellow solids in solution within few minutes to several hours indicated successful synthesis of chalcone. The product was filtered and washed with ice cold water ( 3 $x 10 \mathrm{ml}$ ). The title compound was purified by column chromatography using chloroform, methanol as eluent. The remaining other two substituted 1, 3diarylpropenone were prepared by the similar method.

\subsection{Chemicals and Instruments}

Chemicals and solvents used for the synthesis were procured from Sigma-Aldrich, Spectrochem India Pvt. Ltd and were used without further purification. HPLC grade solvents were used for spectroscopic studies. UV-vis spectra were recorded on Carry 100 Bio Varian EL 0010386 and emission spectra were recorded on a Shimadzu RF-5301 PC Fluorospectrophotometer using cuvette of $1.0 \mathrm{~cm}$ path length. Austin Model 1 (AM1) method was used to calculate the ground state dipole moment.

\section{RESULTS AND DISCUSSION}

\subsection{Preparation of the Compound}

Stock solution of the compound MPPP $\left(1 \times 10^{-3} \mathrm{~mol}\right.$ $\left.\mathrm{L}^{-1}\right)$ in various solvents viz. Methanol, acetone, acetonitrile, 1, 4-dioxane, THF, DMF, DMSO, carbon tetrachloride and ethyl acetate were prepared for all spectral studies. All solvents were checked for any emission in the desired wavelength before fluorescence studies.

\subsection{Effect of Solvents on the Absorption and Fluorescence Spectra}

The UV-visible absorption and fluorescence spectra of MPPP were analyzed in various non polar, polar and neutral organic solvents viz. acetonitrile, ethyl acetate, carbon tetra chloride, THF, DMF, DMSO, acetone, methanol, 1,4-dioxane, and the corresponding spectroscopic data are collected in Table 1. For UVVisible absorption and fluorescence maxima measurements, the concentration was taken $1 \times 10^{-6} \mathrm{M}$ in all solvents.

\subsubsection{Absorption Spectra}

We recorded the absorption spectra of MPPP in neutral, polar and hydrogen bonding organic solvents (Figure 2) and spectroscopic values are summarized in Table 1. It is apparent from the absorption spectra that the compound MPPP in all solvents except in THF showed two absorption bands in the region of $\sim 250$ $297 \mathrm{~nm}$ with higher intensities and $314-393 \mathrm{~nm}$ with lower intensities respectively while the same compound showed single band at $314 \mathrm{~nm}$ in THF. These absorption spectra of MPPP in different solvents attribute the charge transfer from pyrrolidine moiety to

Table 1: Absorbance and Emission Parameters of MPPP in Different Organic Solvents

\begin{tabular}{|c|c|c|c|c|c|}
\hline Solvents & $\lambda \operatorname{abs}(\mathrm{nm})$ & $\lambda \mathrm{flu}(\mathrm{nm})$ & $\operatorname{vabs}\left(\mathrm{cm}^{-1}\right)$ & $v$ flu $\left(\mathrm{cm}^{-1}\right)$ & $\mathrm{v}\left(\mathrm{cm}^{-1}\right)$ \\
\hline Dioxane & 368 & 466 & 27173 & 21459 & 5714 \\
\hline $\begin{array}{l}\text { Carbon Tetra } \\
\text { Chloride }(\mathrm{CCl} 4)\end{array}$ & $297,348,375$ & 460,455 & 28735 & 21739,21978 & 6996,6757 \\
\hline Tetra Hydro Furan (THF) & 314 & 468 & 31847 & 21367 & 10480 \\
\hline Acetonitrile & $296,368,379$ & 481 & 27173 & 20790 & 6383 \\
\hline Ethylacetate & 378 & 469 & 26455 & 21321 & 5134 \\
\hline Methanol & 395 & 484 & 25316 & 20661 & 4655 \\
\hline Acetone & 378 & 472 & 26455 & 21186 & 5269 \\
\hline DMF & 248,387 & 467 & 25839 & 21413 & 4426 \\
\hline DMSO & 301,393 & 476 & 25445 & 21008 & 4437 \\
\hline
\end{tabular}


$\alpha, \beta$ unsaturated carbonyl in the ground state. It is documented that absorption maxima of lower wavelength such as $\sigma \rightarrow \sigma^{*}$ transition with higher intensities is independent of solvent polarity while higher wavelength (lower energy) absorption maxima such as $\pi \rightarrow \pi^{*}$ and $n \rightarrow \pi^{*}$ (with lower intensities) depend on solvent polarity as well as hydrogen bonding efficiency of solvent [22]. Absorption spectrum of MPPP in methanol showed two bands viz. longer wavelength (LW) with a maximum at $\sim 393 \mathrm{~nm}$ and a shorter wavelength band (SW) at $\sim 248 \mathrm{~nm}$. The SW band is ascribed as $\pi-\pi^{*}$ transition of the pyrrolidine substituted benzoid system whereas the LW bands can be attributed to $\pi$ - $\pi^{*}$ transition of the, unsaturated carbonyl of MPPP [23, 24]. These two bands in absorption spectrum can be assigned to the transition from ground (So) to $S_{2}(\mathrm{Lb})$ and $S_{1}(\mathrm{La})$ states from Platt's notation [25, 26].

Moreover, the compound showed bathochromic shift of higher wavelength absorption band (lower energy) in polar solvent like methanol suggesting the possibility of intermolecular hydrogen bonding in ground state $\left(\mathrm{S}_{0}\right)$ between nitrogen of pyrrolidine, and acidic proton of methanol, thus provides higher stability of the solute solvent complex in the ground state [25]. (Figure 2) Carbon tetrachloride being a non polar solvent cannot accept proton but showed the absorption band at $\sim 375 \mathrm{~nm}$ similar to the polar solvents which have tendency to accept proton. We assume that there must be trace amount of water is present in solvent, resulted intermolecular hydrogen bonding with pyrrolidine nitrogen of the compound [26, 27]. This hydrogen bonding gave rise to bathochromic shifts. Further, solvents like Dioxane, DMF, acetone and ethyl acetate have high hydrogen bond accepting capability than the methanol [27]. Dioxane being less polar solvent showed absorption band at $\sim 368 \mathrm{~nm}$, while absorption band is shifted around $\sim 17$ to $25 \mathrm{~nm}$ in more polar solvent like in DMF (387 nm) and in DMSO $393 \mathrm{~nm})$.

Methanol and DMSO showed absorption band at approximately $\sim 395 \mathrm{~nm}$, though methanol is less polar than DMSO. This anomaly can be explained by the fact that methanol, having intermolecular hydrogen bonding property showed intermolecular hydrogen bonding with pyrrolidine nitrogen as well as carbonyl oxygen, that is responsible for higher stability of the solute-solvent system in ground state resulted shifting in band position.

In THF, MPPP showed single absorption band around $314 \mathrm{~nm}$ because the nature of solvated electrons is significantly different in THF than other polar solvents. This difference is due to the presence of many preexisting attractive solvent cavities that are inherent in THF. Due to the dislike character of THF solvent molecules are unable to pack efficiently, thus crating spatial voids. This difference in absorption maxima of THF can also be explained that there is large conformational disorder due to the different groups is involved in salvation in THF than other solvent [28].
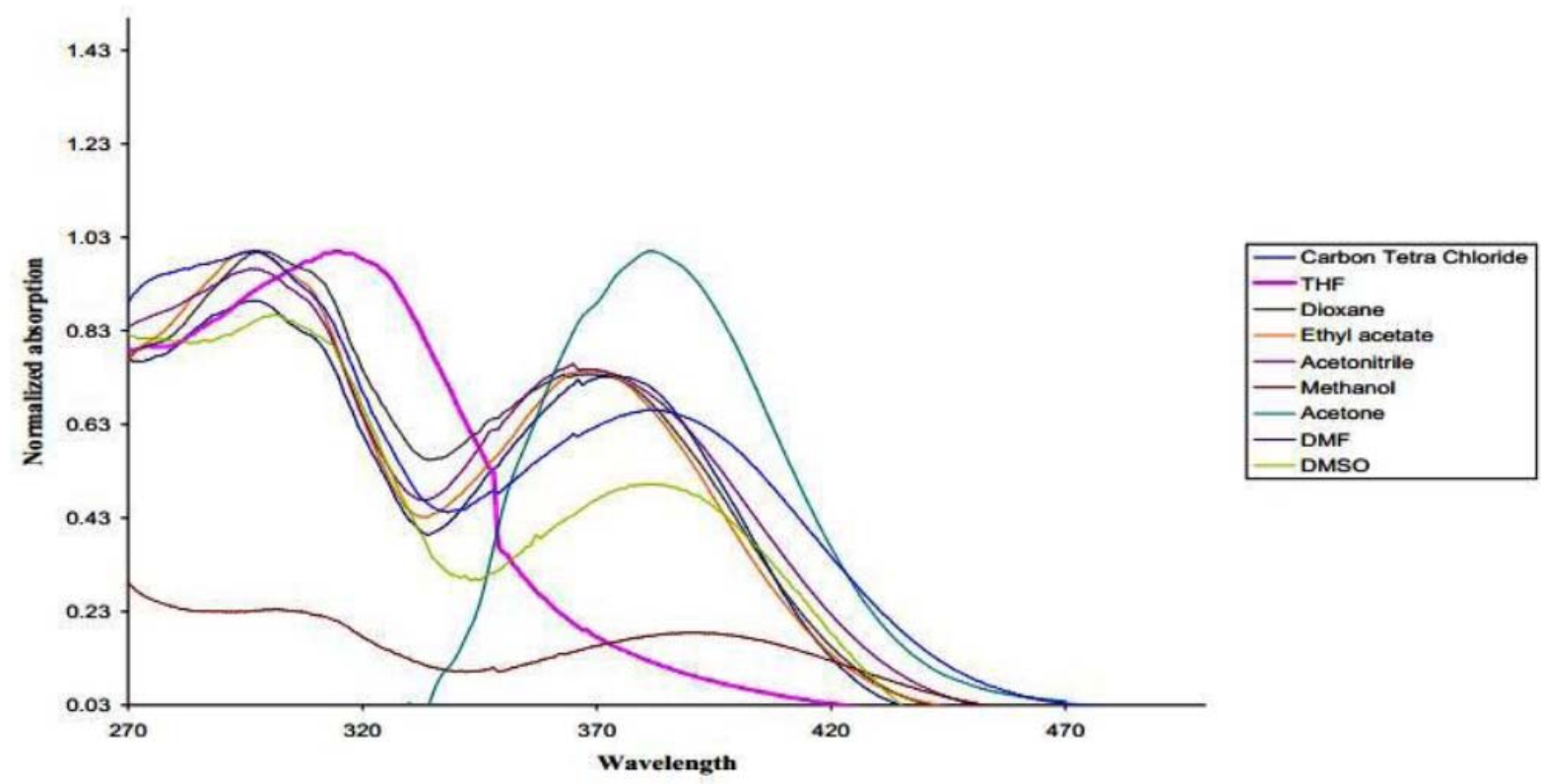

Figure 2: Normalized absorption spectra of MPPP in various solvents; carbon tetra Chloride, Tetrahydrofuran, 1, 4-Dioxane, Ethyl acetate, acetonitrile, $\mathrm{MeOH}$, acetone, DMF and DMSO. 


\subsubsection{Fluorescence Spectra}

We recorded the fluorescence emission spectra of MPPP in various solvents as shown in Figure $\mathbf{3}$ and observed band maxima are listed in Table 1. MPPP showed single emission wavelengths in between 455$484 \mathrm{~nm}$ depending on solvent polarities. Unlike absorption spectra, emission spectrum bands are broad, in the large visible region and more sensitive to solvents which suggest that greater charge transfer in solute-solvent complex takes place in excited state [24].

It is apparent that fluorescence spectrum shows bathochromic shifts as solvent polarity is increased. The red shift in the emission maximum from $455 \mathrm{~nm}$ in carbon tetrachloride to $484 \mathrm{~nm}$ in methanol clearly suggested that MPPP possess significant intramolecular charge transfer resulting in intramolecular polarization in the excited state due to $\pi-\pi^{\star}$ transition, since it contain both electron donor groups such as pyrrolidine group and electron acceptor methoxy group. This suggests that MPPP is significantly solvated in the S1excited state, resulted in a large dipole moment difference between the S1excited state and the ground state [26].

This fluorescence band can be assigned to transition involving the whole electronic system of the compounds with a considerable charge transfer (CT) character. Such a CT originates from the nitrogen to

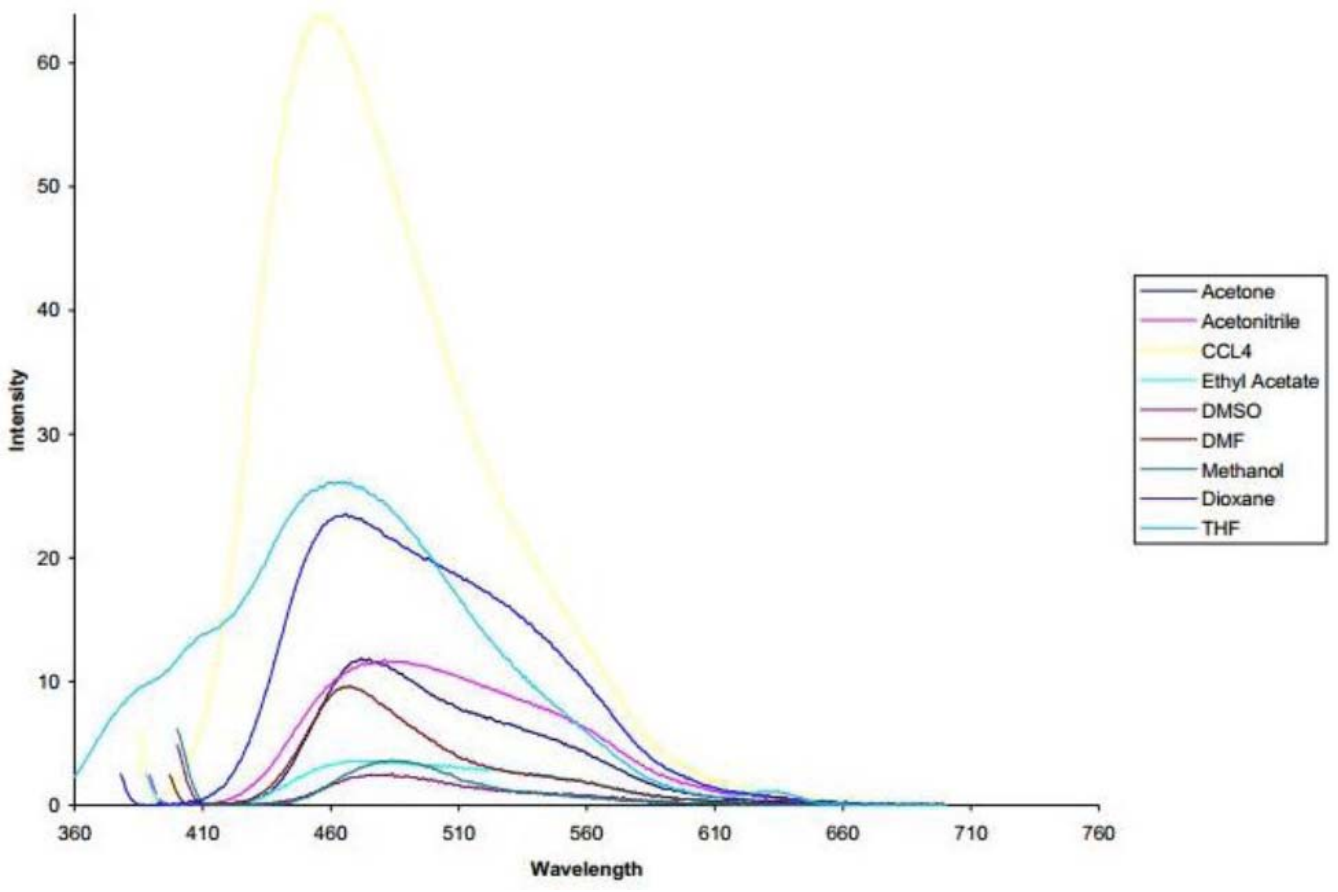

Figure 3: Fluorescence spectra of MPPP in different organic solvents. the carbonyl group which is characterized by a higher electron accepting character, it means this visible band occur due to intramolecular charge transfer (ICT) [22].

A large red shift in the emission band maxima reflects the greater delocalization of the cloud of the carbonyl group and the lone pair of nitrogen of pyrrolidine moiety with the aromatic moiety. In solvents more polar than carbon tetra chloride and ethyl acetate, the emission maxima shift to the longer wavelength (red shift).

The fluorescence intensity is maximum in $\mathrm{CCl}_{4}$ which is a non-polar solvents, thus molecule shows a high intramolecular charge transfer state which is stabilized by polar and non polar solvents. In this case, the donor group is nitrogen of aromatic moiety of the molecule, and the acceptor is carbonyl group similar to that absorption spectra. Contrary to the non hydrogen bonding solvents (Dioxane, THF, EA) the emission spectra of MPPP are more red shifted (484 nm) in hydrogen bonding solvent (methanol) Figure 3 . This change in spectra is due to the formation of donoracceptor complex in alcoholic solution. The fluorescence spectrum in each solvent is broader, that is having a broader full width at half the maximum hight $(F W M H)$. The FMWH of the fluorescence spectra increases in the polar solvents. Comparing with other studied system, we have assigned the lower energy emission band to the emission from the charge transfer (CT) state [25]. It is found that the peak positions of the 
emission bands are independent of excitation wavelength over a large range of absorption band. The excitation spectra of both the bands are independent of emission wavelength and agree reasonable with the absorption spectrum. So we can say that only one species is present in the ground state and the Charge transfer (CT) band is generated through the Lower energy (LE) state.

Unlike the absorption spectra, the fluorescence spectra of MPPP in basic (aprotic) solvent such as dioxane, exhibits dual emission band as shown in Figure 3. The excitation spectra exhibit a broad band at around $\sim 368 \mathrm{~nm}$ and a small shoulder at $\sim 540 \mathrm{~nm}$ indicating the presence of two moieties in ground state. While in other basic solvents like ethyl acetate and DMF, fluorescence spectra showed single broad peak of less intensity unlike two intense bands in absorption spectra. Based on these observations we conclude that only one moiety is active in ground state of MPPP.

A qualitative analysis of the red shift of lower energy emission band indicated that the emissive species must possess high dipolar character [10]. In methanol, the emission maxima was maximum and markedly shifted to the red region than the other solvents, this indicate that MPPP's excited state is more polar than the ground state.

\subsection{Stokes Shift}

The fluorescence spectrum was exploited to establish correlation between peak wave numbers of fluorescence spectra and the empirical Dimorth polarity parameter $E_{T}$ (30) of the solvents [14] as shown in Figure 4 and calculated $E_{T}(30)$, normal $E_{T}$ and fluorescence quantum yield shown in Table 2. Figure 4 shows excellent linear correlation in case of solvent like DMSO, ethyl acetate, acetone, dioxane. However, a little deviation from linearity of Stokes shifts versus $\Delta f$ in protic solvents like methanol; acetonitrile and THF were also seen. The maximum deviation was observed in DMF. This suggested that some additional factors affecting the system, thus making it non-linear.

In general, protic solvents have a nature to form intermolecular hydrogen bonded complex and the weak hydrogen bonding interaction significantly influences the properties in the ground state as well as in the excited state.

In earlier studies [29], it was explained that aromatic amines present in the molecules show a large departure from the linearity for the protic solvents due to the formation of hydrogen bond between protic solvents and lone pair electron of amino group in $\mathrm{S}_{0}$ state, but in excited state this bond break down due to

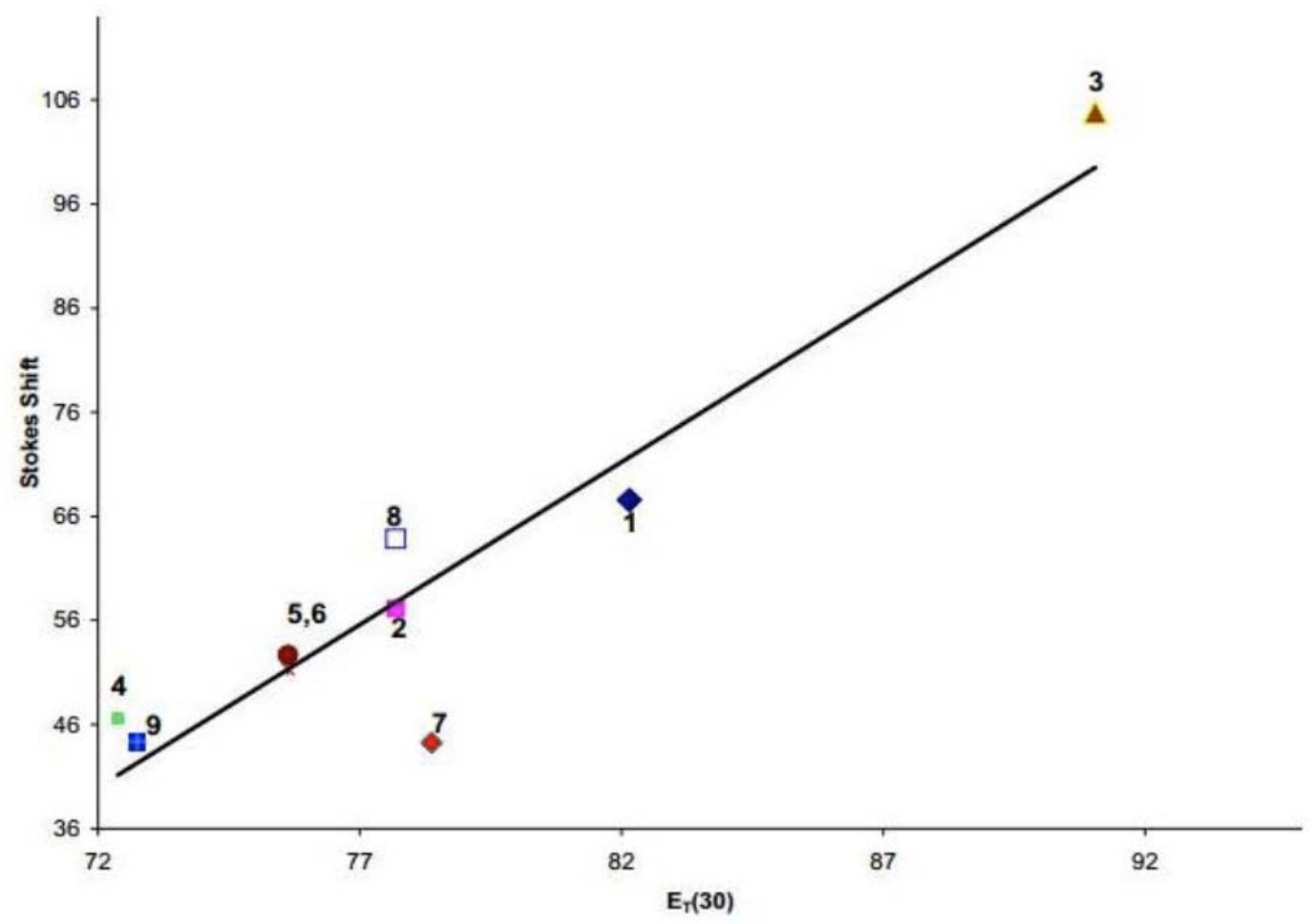

Figure 4: Graph between peak wave number $(\Delta v)$ and Dimorth Polarity parameter ET (30); (1) carbon tetra Chloride, (2) 1, 4Dioxane, (3) Tetrahydrofuran, (4) MeOH, (5) Ethyl acetate, (6) acetone, (7) DMF, (8) Acetonitrile and (9) DMSO. 
Table 2: Dimorth Polarity Parameter and Fluorescence Quantum Yield

\begin{tabular}{|c|c|c|c|c|}
\hline Solvents & $\mathbf{E}_{\mathbf{T}}(\mathbf{3 0})$ & $\mathbf{E}_{\mathbf{T}}$ (normal) & Of & \multicolumn{1}{c|}{0.50} \\
\hline \hline Dioxane & 77.69 & 77.48 & 0.0294 \\
\hline $\begin{array}{c}\text { Carbon Tetra } \\
\text { Chloride (CCl4) }\end{array}$ & 82.15 & 82.09 & 0.0112 \\
\hline $\begin{array}{c}\text { Tetra Hydro } \\
\text { Furan (THF) }\end{array}$ & 91.05 & 90.85 & 0.087 & 0.2088 \\
\hline Acetonitrile & 77.69 & 77.48 & 0.02 & 0.3049 \\
\hline Ethyl acetate & 75.63 & 75.63 & 0.004 & 0.1996 \\
\hline Methanol & 72.38 & 72.17 & 0.019 & 0.3088 \\
\hline DMF & 78.37 & 73.79 & 0.025 & 0.2752 \\
\hline DMSO & 72.75 & 72.63 & 0.029 & 0.2632 \\
\hline
\end{tabular}

the formation of reverse hydrogen bonding i.e., between amino proton and lone pair of protic solvent. This straight line formation in case of $E_{T}$ (30) parameters reflects a dispersive and specific interaction in these parameters [30]. Non linearity in case of solvents like DMF, acetonitrile, THF and methanol showed deviation.

The linear correlation between $E_{T}$ values and Stokes shift $(\Delta v)$ are in good agreement with an extensive intramolecular charge transfer upon excitation of the molecule. Moreover, polarizability scale can also be represented as a function of refractive index i.e. $\left(n^{2}-1\right) /\left(2 n^{2}+1\right)$ [31] as shown in
Figure 5. Thus electron redistribution of electrons generates the spectral shift Figure 5 . The graph shows that MPPP possesses a strong intramolecular charge transfer by increasing polarization in its excited state.

\subsection{Fluorescence Quantum Yield}

Compound showed fluorescence in all studied solvents, the intensity was maximum in acetone. The fluorescence quantum yields $\left(\phi_{s}\right)$ of MPPP in solvent of different polarities were calculated using the following equation [32]. 2-Amino pyridine in $0.1 \mathrm{M} \mathrm{H}_{2} \mathrm{SO}_{4}$ was used as standard reference, $\phi_{r}=0.60$ [33]

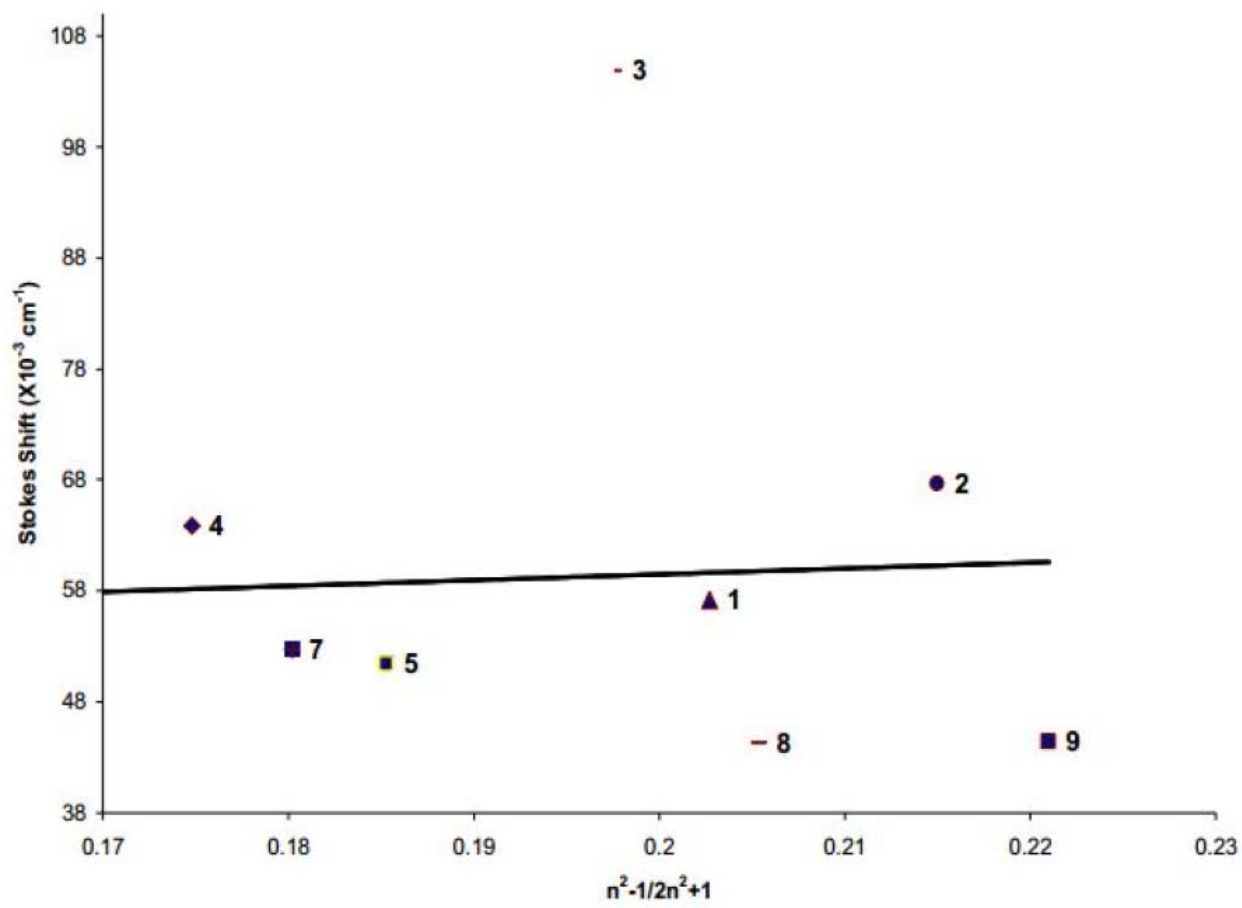

Figure 5: Graph between peak wave number $\Delta v(x$ 10-3cm-1) and index of refraction; (1) 1, 4-Dioxane, (2) carbon tetra Chloride, (3) Tetrahydrofuran, (4) Acetonitrile. 


$$
\phi_{s}=\left[\left(A_{r} F_{s} n_{s}^{2} /\left(A_{r} F_{s} n_{s}^{2}\right)\right] \phi_{r}\right.
$$

Where $A$ is absorbance at the excitation wave length, $\mathrm{F}$ the integrated emission area and $\mathrm{n}$ the refraction index of the solvents used. Subscripts refer to the reference ( $r$ ) or sample (s) compound.

The fluorescence quantum yields $\left(\phi_{s}\right)$ depends strongly on the solvent polarity and the acceptor strength of the keto-phenyl group (Table 2) [26].

MPPP shows significant quantum yield $\left(\phi_{s}\right)$ which depends on solvent polarity, acidity and basicity of the solvents as shown in Table 2. Figure 6 represents a relationship between $\phi_{s}$ and the solvent polarity parameter $\mathrm{E}_{\mathrm{T}}(30)$. It is clear from the Figure 6 that the fluorescent quantum yield increases with the increasing the solvent polarity from $\mathrm{CCl}_{4}$ to dioxane, reaching to its maximum in 1, 4 dioxane. Further, increase in solvent polarity leads to a dramatic decrease in quantum yield, which is very less in case of ethyl acetate. As we know that solvent ethyl acetate, DMF and acetone have high bond accepting capability, thus these solvents can either accept or donate hydrogen from pyrrolidine nitrogen of MPPP. A very efficient electron transfer from ethyl acetate to MPPP in excited state is responsible for the very low $\phi_{s}[14]$.

Based on previous photophysical studies, two mechanisms had been proposed for substituted chalcones to explain the increase or decrease in quantum yield of fluorescent compound by changing the solvent polarity [34]. The "Negative solvatokinetic effect" explains an increase in the fluorescence yield $s$ with a suitable enhancement of the ICT character. Several suggestions have been given such as, 'proximity effect', 'biradicaloid charge transfer' and 'conformational changes' to explain 'Negative solvatokinetic effect". Thus, solute-solvent complexes formed in the ground state results in an effective nonradiative loss and quenching of the MPPP fluorescence occur in excited state due to hydrogen bond interaction [35]. Further, a decrease in the fluorescence quantum yield by strong ICT falls under "Positive solvatokinetic effect". Thus, both "Negative and Positive solvatokinetic effect" have significant contributions on MPPP fluorescence quantum yields. Therefore, as solvent polarity changes from $\mathrm{CCl}_{4}$ to 1 , 4-dioxane, fluorescence quantum yields increases (0.32 to 0.50 ) as it is governed by "Negative solvatokinetic effect. However, in solvent with higher polarity from THF to methanol, the fluorescence quantum yields decreases (0.087 to 0.019$)$ due to strongly intramolecular charge transfer (ICT), i.e. "Positive solvatokinetic effect". It can be attributed from second mechanism that a strong ICT interaction occurs between solute and solvent [34].

\subsection{Solvatochromasim}

The UV-vis and fluorescence spectral results of MPPP in various organic solvents are summarized in

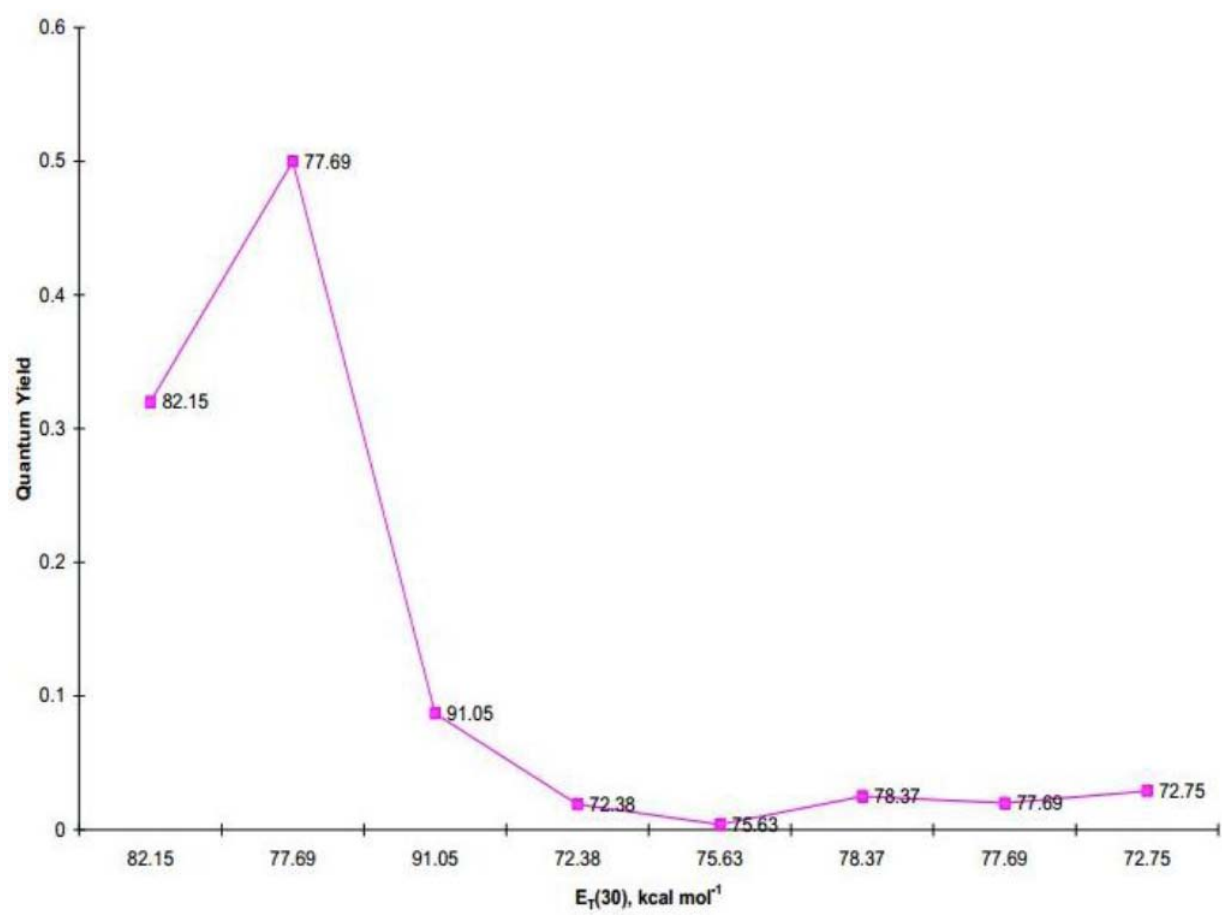

Figure 6: Change in fluorescence quantum yield $(\phi)$ of MPPP with the Dimorth solvent polarity parameter. 
Table 1. Solvatochromic behaviour of compound MPPP in ground and excited state helps to determine the change in dipole moment upon excitation $\left(\mu^{*}\right)$ using Lippert-Mataga equation [25].

$v_{a}-v_{f}=\left[2 / h c a^{3}\right] \Delta f\left(\mu^{*}-\mu\right)^{2}$

$\Delta f=[\varepsilon-1 / 2 \varepsilon+1]-\left[n^{2}-1 / 2 n^{2}+1\right]$

Where, $\mathrm{h}$ is Planck's constant, $\mathrm{c}$ is the speed of light, and $a$ is Onsager cavity radius, $\varepsilon$ and $n$ are the dielectric constant and refractive index of the solvent, respectively. The absorption and emission frequencies are va and vf respectively. $(\varepsilon-1) /(2 \varepsilon+1)$ expresses the spectral shifts produced by two factor namely the solvent dipole reorientation and electron redistribution in solvent molecules while $(n 2-1) /\left(2 n^{2}+1\right)$ expresses the spectral shifts due to electron redistribution. The difference between these two items represents the spectral shifts caused by the solvent molecular reorientation. This is shown in the plot of Stoke shift and solvent parameter $(\Delta f)$ Figure 7. A linear relationship between the Stokes shifts and solvent parameters in aprotic solvents predicts large dipole moment of the emissive species. The ground $(\mu)$ and excited state $\left(\mu^{*}\right)$ dipole moment of MPPP was calculated using solvatochromic method and was found 5.58 and $5.90 \mathrm{D}$ respectively. The change in dipole moment from the ground state to excited state suggested that the charge transfer occurs through the relaxation of ground state to excited state. Thus, the solvent dependent emission shifting was observed. $\Delta f$ $=\left[\begin{array}{ll}\varepsilon-1 / 2 & \varepsilon+1\end{array}\right]-\left[n^{2}-1 / 2 n^{2}+1\right]$ a function which is independent of polarisibility effect. Further, Dipole moment $\left(\mu^{*}\right)$ of excited state was calculated from spectroscopic values using the following equation [36].

$\left(\mu^{*}-\mu\right)=\Delta \mu=\sqrt{ } m \times 81 /(6.2 / a)^{3} \times 11307.6$

Where 'a' is Onsager cavity radius. $\Delta \mu$ is extracted from the slope $(\mathrm{m})$ of the plot of stokes shift versus $E_{T}^{N}$. The value of Onsager cavity radius (a) was calculated from the following equation (5) [22],

$a={ }^{3} \sqrt{ } 3 M / 4 \pi d N$

Here, $\mathrm{M}$ is molecular weight of the compound, $\mathrm{N}$ is Avogadro number, and $d$ is the density $\left(d=0.15 \mathrm{~g} / \mathrm{cm}^{3}\right)$. By using the Eq. 5, Onsager cavity radius was calculated to be $8.55 \AA$. Thus, using calculated $\Delta \mu$, dipole moment of MPPP molecule in the excited state was calculated.

\section{CONCLUSION}

Photoinduced charge transfer in MPPP using various solvents is discussed. It is apparent from the

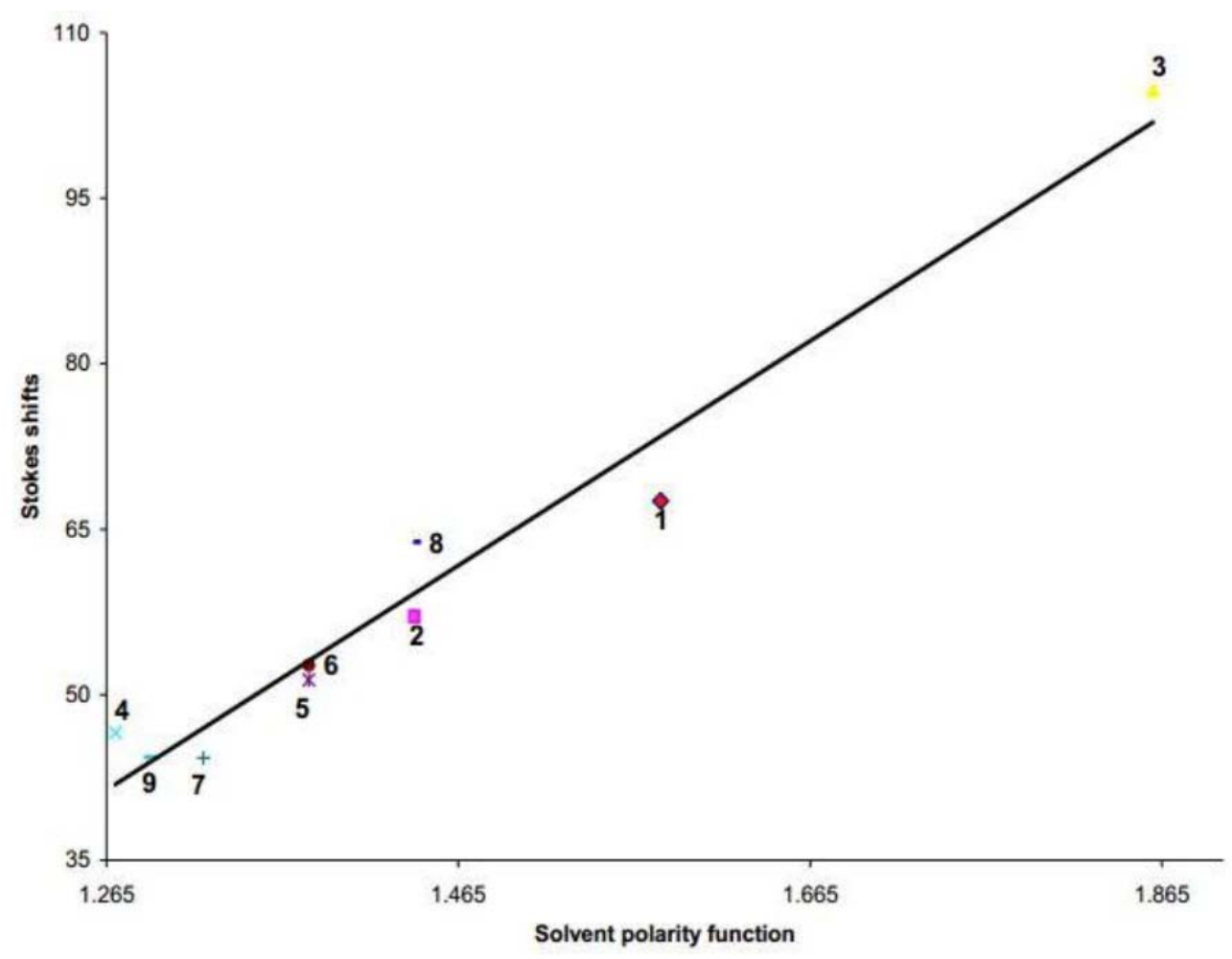

Figure 7: Graph between Stokes shift and solvent polarity function $(\Delta \mathrm{f})$ of MPPP; (1)1, 4-Dioxane, (2) carbon tetra Chloride, (3) Tetrahydrofuran, (4) Acetonitrile (5) Ethyl acetate, (6) MeOH, (7) acetone, (8) DMF and (9) DMSO. 
experiments that MPPP is very sensitive to the environment polarity due to intramolecular charge transfer (ICT). Moreover, due to the high fluorescence quantum yields, MPPP may be used to probe the solvent polarity and hydrogen bonding properties as well as its microenvironment in biological macromolecules of interest.

\section{ACKNOWLEDGEMENT}

Nidhi Mishra is thankful to Department of Science and Technology (DST) New Delhi for the financial support and Planaria Software LLC for their kind license to use ArgusLab software.

\section{REFERENCES}

[1] Clark RL, Pessonlado AA, Witzal B, et al. 2-(Substituted phenyl)oxazolo[4,5-b]pyridines and 2-(substituted phenyl)oxazolo[5,4-b]pyridines as nonacidic antiinflammatory agents. J Med Chem 1978; 21: 1158-1162. http://dx.doi.org/10.1021/jm00209a014

[2] Lackwicz RJ. Topics in Fluorescence Spectroscopy: Probe, Design, and Chemical Sensing, Plenum Press: New York 1994; 4: 295-334.

[3] Wang P, Wu S. Spectroscopy and photophysics of bridged enone derivatives: effect of molecular structure and solvent. J Photochem Photobiol A: Chem 1985; 86: 109-113. http://dx.doi.org/10.1016/1010-6030(94)03921-G

[4] Rotkiewiez K, Grellmann KH, Grabowski ZR. Reinterpretation of the Anomalous Fluorescence of $\mathrm{p}-\mathrm{N}, \mathrm{N}$-Dimethylaminobenzonitrile. Chem Phys Lett 1973; 19: 315-318. http://dx.doi.org/10.1016/0009-2614(73)80367-7

[5] Lin YM, Zhou Y, Flavin MT, Zhou LM, Nie W, Chen FC. Chalcones and flavonoids as anti-tuberculosis agents. Bioorg Med Chem 2008; 10: 2795-2802.

http://dx.doi.org/10.1016/S0968-0896(02)00094-9

[6] Sun SJ, Schwarzm G, Kricheldorf RH, Chang TC. New polymers of carbonic acid. XXV. Photoreactive cholesteric polycarbonates derived from 2, 5-bis(4'-hydroxybenzylidene) cyclopentanone and isosorbide. J Polym Sci A: Polym Chem 1999; 37: 1125-1133.

http://dx.doi.org/10.1002/(SICI)10990518(19990415)37:8<1125::AID-POLA9>3.0.CO;2-J

[7] Sato $Y$, Morimoto $M$, Segawa $H$, Shimidzu T. Twisted Intramolecular Charge-Transfer State of a Donor-Acceptor Molecular System with a $\beta$-Diketone Bridge: Tuning of Emission through Structural Restriction by Metal Cation Coordination. J Phys Chem 1995; 99: 35-39.

http://dx.doi.org/10.1021/j100001a007

[8] Marcotte N, Fery-Forgues S, Lavabre D, Marguet S, Pivovarenko VG. Spectroscopic Study of a Symmetrical Biscrown Fluoroionophore of the Diphenylpentadienone Series. J Phys Chem A 1999; 103: 3163-3170.

http://dx.doi.org/10.1021/jp9846328

[9] DiCesare N, Lakowicz JR. Chalcone-analogue fluorescent prfobes for saccharides signaling using the boronic acid group. Tetrahedron Lett 2002; 43: 2615-2618. http://dx.doi.org/10.1016/S0040-4039(02)00312-X

[10] Xu Z, Bai G, Dong C. Determination of human serum albumin using an intramolecular charge transfer fluorescence probe: 4'-Dimethylamino-2, 5-dihydroxychalcone. Bioorg Med Chem Lett 2005; 15: 4091-4096.

http://dx.doi.org/10.1016/j.bmcl.2005.06.014
[11] Shannigrahi M, Bagchi S. Novel fluorescent probe as aggregation predictor and micro-polarity reporter for micelles and mixed micelles. Spectrochim Acta A 2005; 61: 21312138.

http://dx.doi.org/10.1016/j.saa.2004.08.012

[12] Das PK, Pramanik R, Banerjee D, Bagchi S. Studies of solvation of ketocyanine dyes in homogeneous and heterogeneous media by UV/Vis spectroscopic method. Spectrochim Acta A 2000; 56: 2763-2773. http://dx.doi.org/10.1016/S1386-1425(00)00321-8

[13] Xu Z, Bai G, Dong C. Spectral and photophysical properties of intramolecular charge transfer fluorescence probe: 4'Dimethylamino-2, 5-dihydroxychalcone. Spectrochim Acta A 2005; 62: 987-990. http://dx.doi.org/10.1016/j.saa.2005.04.019

[14] Wang P, Wu S. Spectroscopy and photophysics of bridged enone derivatives: effect of molecular structure and solvent. J Photochem Photobiol A: Chem 1995; 86: 109-113. http://dx.doi.org/10.1016/1010-6030(94)03921-G

[15] Jiang YB, Wang XJ, Lin L. Fluorescent Probing of the Restriction by Aqueous Micelles of the Formation of the Photoinduced Biradical State $P^{*}$ of 4-(Dimethylamino) chalcone. J Phys Chem 1994; 98: 12367-12372. http://dx.doi.org/10.1021/j100098a035

[16] Rurack K, Bricks JK, Reck G, Radegha R, Resch-Genga U. Chalcone-Analogue Dyes Emitting in the Near-Infrared (NIR): Influence of Donor-Acceptor Substitution and Cation Complexation on Their Spectroscopic Properties and X-ray Structure. J Phys Chem A 2000; 104: 3087-3109. http://dx.doi.org/10.1021/jp994269k

[17] Eisenhart JM, Ellis AB. Perturbation of the excited-state properties of trans, trans-1, 5-bis[4-(dimethylamino)phenyl]1,4-pentadien-3-one through adduct formation and silica gel adsorption. J Org Chem 1985; 50: 4108-4113.

http://dx.doi.org/10.1021/jo00221a028

[18] Doroshenko AO, Grigorovich AV, Porsokhov EA, Pivovarenko VC, Demchenko AP. Bis-Azacrown Derivative of Di-Benzylidene-Cyclopentanone as Alkali Earth Ion Chelating Probe: Spectroscopic Properties, Proton Accepting ability and Complex Formation with $\mathrm{Mg} 2+$ and $\mathrm{Ba} 2+$ lons. Mol Eng 1999; 8: 199-215.

http://dx.doi.org/10.1023/A:1008393201193

[19] Mishra N, Arora P, Kumar B, et al. Synthesis of novel substituted 1, 3-diaryl propenone derivatives and their antimalarial activity in vitro. Eur Med Chem 2008; 43: 15301535. http://dx.doi.org/10.1016/i.ejmech.2007.09.014

[20] Awasthi SK, Mishra N, Kumar B, et al. Potent Antimalarial Activity of Newly Synthesized Substituted Chalcone Analogs in vitro. Med Chem Res 2009; 18: 407-420. http://dx.doi.org/10.1007/s00044-008-9137-9

[21] Bansal PR, Lahiri S, Kar S, Chakravorti S. Vibronic interaction and photophysics of chalcone derivatives. J Luminsence 1996; 69: 49-56. http://dx.doi.org/10.1016/0022-2313(96)00035-X

[22] Stalin T, Rajendiran R. Intramolecular charge transfer associated with hydrogen bonding effects on 2-aminobenzoic acid. J Photochem Photobiol A: Chem 2006; 182: 137-150. http://dx.doi.org/10.1016/j.jphotochem.2006.02.002

[23] Platt JR. Classification of spectra of Cata-Condensed Hydrocarbons. J Chem Phys 1949; 17: 484-495. http://dx.doi.org/10.1063/1.1747293

[24] Khalaf AA, Etaiw SH, Issa RM, El-Shafei AK. Spectrophotometric study of some 1, 5-diphenyl-1,5pentadiene-3-ones. Rev Roum Chim 1977; 22: 1251-1259.

[25] Chakraborty A, Kar S, Guchhait N. Secondary amino group as charge donor for the excited state intramolecular charge transfer reaction in trans-3-(4-monomethylamino-phenyl)acrylic acid: Spectroscopic measurement and theoretical 
calculations. J Photochem Photobiol A: Chem 2006; 181: 246-256.

http://dx.doi.org/10.1016/j.jphotochem.2005.12.004

[26] Fayed TA. A novel chalcone-analogue as an optical sensor based on ground and excited states intramolecular charge transfer: A combined experimental and theoretical study. Chem Phys 2006; 324: 631-638.

http://dx.doi.org/10.1016/i.chemphys.2005.11.039

[27] Frade VHJ, Goncalves MST, Coutinho PJG, Moura JCVP. Synthesis and spectral properties of long-wavelength fluorescent dyes. J Photochem Photobiol A: Chem 2007; 185: 220-230.

http://dx.doi.org/10.1016/j.jphotochem.2006.06.013

[28] Hearn MJB, Larsen RE, Schwartz BJ. The Role of Solvent Structure in the Absorption Spectrum of Solvated Electrons: Mixed Quantum/Classical Simulations in Tetrahydrofuran (THF). J Chem Phys 2005; 122: 134506- 1-11.

[29] Mazumdar S, Anoharan RM, Dogra SK. Solvatochromic effects in the fluorescence of a few diamino aromatic compounds. J Photochem Photobiol A: Chem 1989; 46: 301314.

http://dx.doi.org/10.1016/1010-6030(89)87047-9

[30] Dogra SK. Spectral characteristics of 2-(3', 5'-diaminophenyl) benzothiazole: effects of solvents and acid-base concentrations. J Photochem Photobiol A: Chem 2005; 172: 185-195.

http://dx.doi.org/10.1016/.jphotochem.2004.12.008

[31] Xin Y, Shen G-L, Yu R-Q. Studies on Intramolecular Charge Transfer Fluorescence Probe and DNA Binding Characteristics. Microchem J 1999; 62: 394- 404. http://dx.doi.org/10.1006/mchj.1999.1747

[32] Demas JN, Crosby GA. Measurement of photoluminescence quantum yields. Rev J Phys Chem 1971; 75(8): 991-1024. http://dx.doi.org/10.1021/j100678a001

[33] Rusakowicz R, Testa AC. 2-Aminopyridine as a standard for low-wavelength spectrofluorimetry. J Phys Chem 1968; 72 : 2680-1.

http://dx.doi.org/10.1021/j100853a084

[34] Reichardt C. Empirical Parameters of Solvent Polarity as Linear Free-Energy Relationships. Angew Chem Int Ed Engl 1979; 18: 98-110. http://dx.doi.org/10.1002/anie.197900981

[35] Mataga N, Kubota T. Molecular Interactions and Electronic Spectra. Marcel Dekker: New York 1970; pp. 371-410.

[36] Reichardt C. Solvents and Solvent Effect in Organic Chemistry. $2^{\text {nd }}$ ed. WILEY-VCH Verlag $\mathrm{GmbH} \&$ Co. KGaA: Weinheim 1988. 